\title{
Design and Implementation of Solar Tracking System
}

\author{
Liping Dong ${ }^{1, \mathrm{a}}$, Zhen $\mathrm{An}^{2, \mathrm{~b}}$, Lina Hao ${ }^{3, \mathrm{c} *}$
}

${ }^{1,2,3}$ School of Mechanical Engineering and Automation, Northeastern University, Shenyang, China

110000

a 850876907@qq.com

b981221164@qq.com

chaolina@me.neu.edu.cn

Keywords: solar energy; tracking system; work efficiency; stability

\begin{abstract}
In order to improve the performance of the tracking system, this paper discusses the trajectory tracking of the sun and tracing to the source of the sun. The experimental results show that: The automatic tracing of sun trajectory scheme runs smoothly without the interference of light source, but has model error; the automatic tracking of the sun source scheme has better accuracy, but the system may be disorder due to the insufficient light intensity .The paper make the two schemes together at the end, so that the tracking system can have better stability and accuracy.
\end{abstract}

\section{Introduction}

According to theoretical analysis, automatic tracking device's energy acceptance rate is $30 \%-40 \%$ higher than that of the fixed chasing equipment so it is necessary and makes sense to equip automatic tracking system in solar energy collection device [1, 2]. This paper uses two kinds of tracking scheme and carries on the comparative analysis. Two kinds of scheme adopt the same mechanical structure, controller, motor drive circuit and DC motor. The mechanical structure of solar energy tracking system has two mutually perpendicular axes, the lower motor rotate in the horizontal direction to reflect the change of the sun azimuth; the upper one rotate in vertical direction to reflect the change of solar altitude angle. Two motor orthogonal motions realize the tracking of the sun. The mechanical structure is shown in Figure 1.

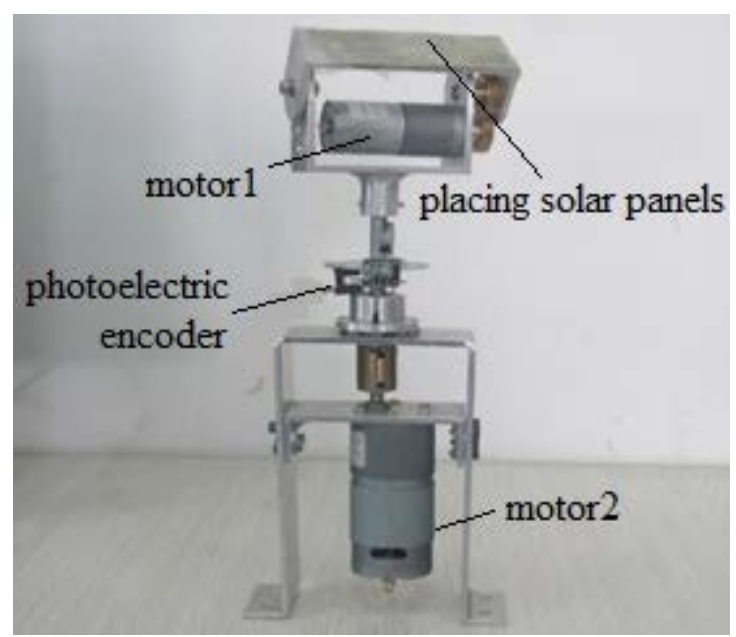

Fig. 1 mechanical structure

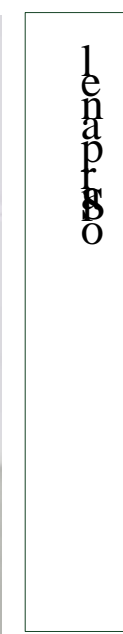

Fig. 2 hardware structure

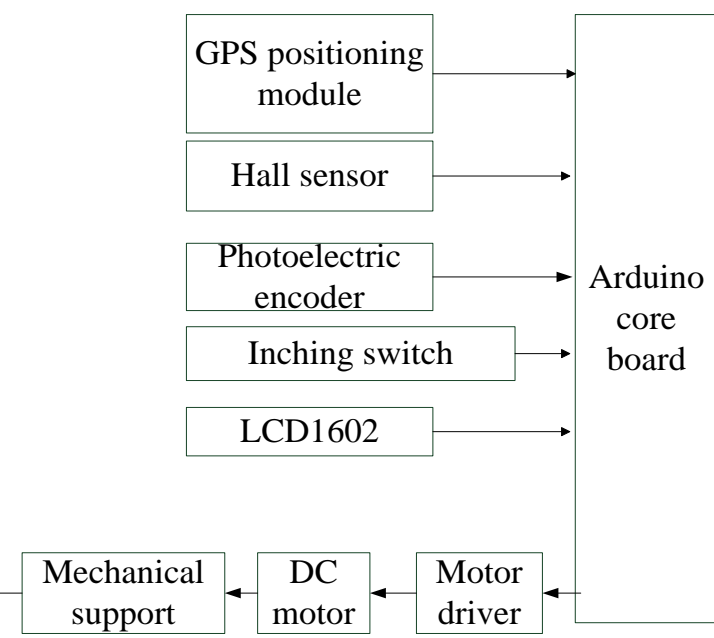

\section{The automatic tracking of the sun trajectory}

The design is "to the sun trajectory tracking". To achieve our goal, we decided as follows: the core board of arduino mega2560 is used as the controller, hall sensors is used as the systemic 
compass [3], the GPS module of U-BLOX chip is used to obtain the latitude, longitude and time information of the system [4,5], photoelectric encoder is used to make the DC motor rotate precisely, LCD1602 liquid crystal is used to display the information of the tracking system about time, position and direction for facilitating debugging, the drive circuit chip of L298N is used to drive DC motor [6], ASLONG JGA25-370 (12V-24RPM) and ASLONG JGB37-545 (24V-35RPM) DC motor are used as the actuator of the system. The hardware structures of the system are shown in Figure 2 and the working procedure is shown in Figure 3.

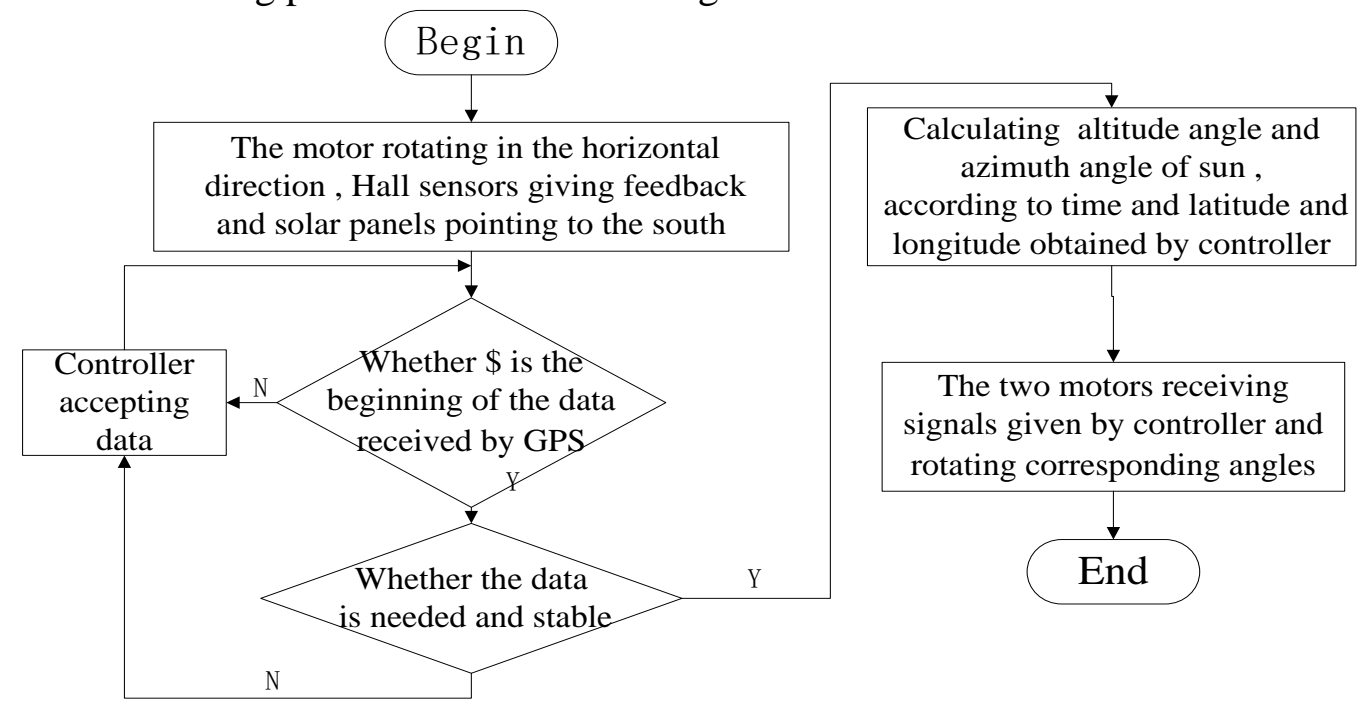

The mathematical modeling of the tracking system

The angle between the sun-earth line and the earth's equatorial plane is the declination angle, the calculation formula of the declination angle, solar altitude angle and azimuth angle are shown as follows:

$$
\begin{aligned}
& \delta=23.45 \times \sin [2 \pi \times(284+n) / 365] \\
& \sin h=\sin \varphi \sin \delta+\cos \varphi \cos \delta \cos t \\
& \sin \gamma=\cos \delta \sin t / \cos h
\end{aligned}
$$

Where $n$ is the number of days, $\delta$ is the declination angle, $h$ is the solar altitude angle, $\gamma$ is the sun azimuth, $\delta$ is the declination angle, $\varphi$ is the geographic latitude, $t$ is the sun hour angle.

Assuming that the solar energy equipment lies in coordinate origin $\mathrm{O}$ in the surface of the earth, the normal direction of verticals upward is regarded as the Z-axis, the direction towards the geographic south pole is regarded as $\mathrm{X}$-axis, and the direction of east is regarded as Y-axis and a left-handed coordinate system is composed of these three axis. OK is the vertical line of solar panels, $\mathrm{S}$ is the position of the sun, when the $\mathrm{O}, \mathrm{S}, \mathrm{K}$ three points are collinear, solar panels is facing the sun. The corresponding coordinate system is shown in Figure 4 and the schematic diagram of optical system is shown in Figure 5.

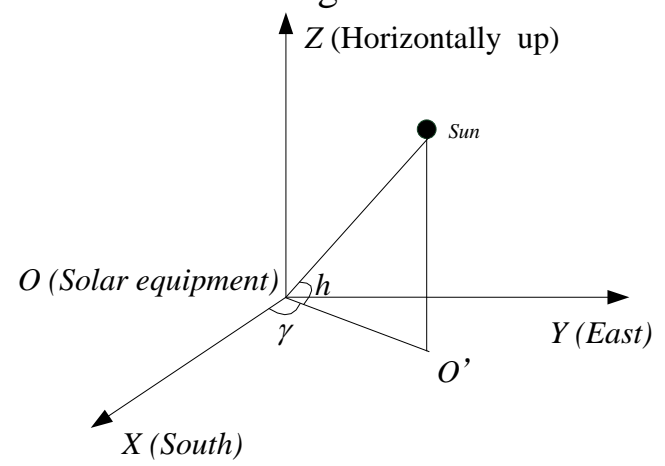

Fig. 4 solar power equipment coordinate system

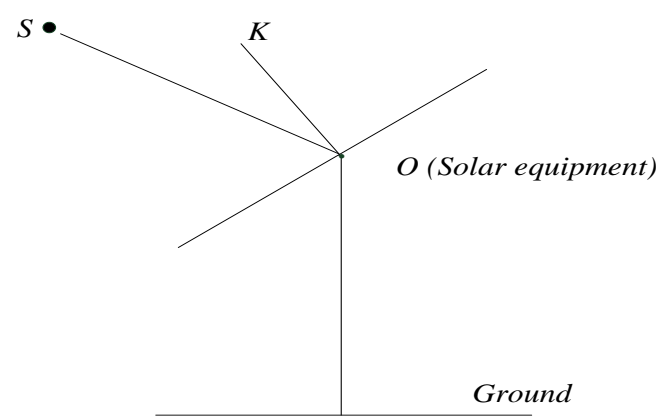

Fig. 5 the schematic diagram of optical system 


\section{The automatically tracking to the source of the sun}

The design idea is "to automatically track the sun light". To develop the following programs, the arduino mega 2560 core board is used as a system controller [7] , 3DU5C phototransistor is used as a photoelectric sensor .System hardware structure is shown in Figure 6 and the working procedure is shown in Figure 7.

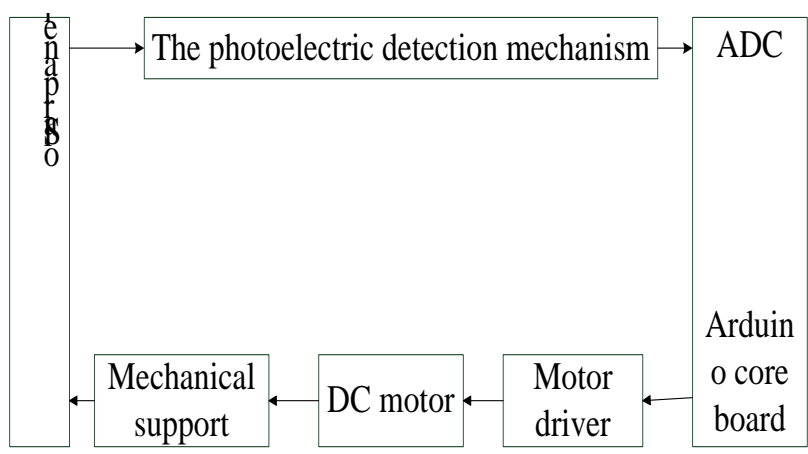

Fig. 6 hardware structure

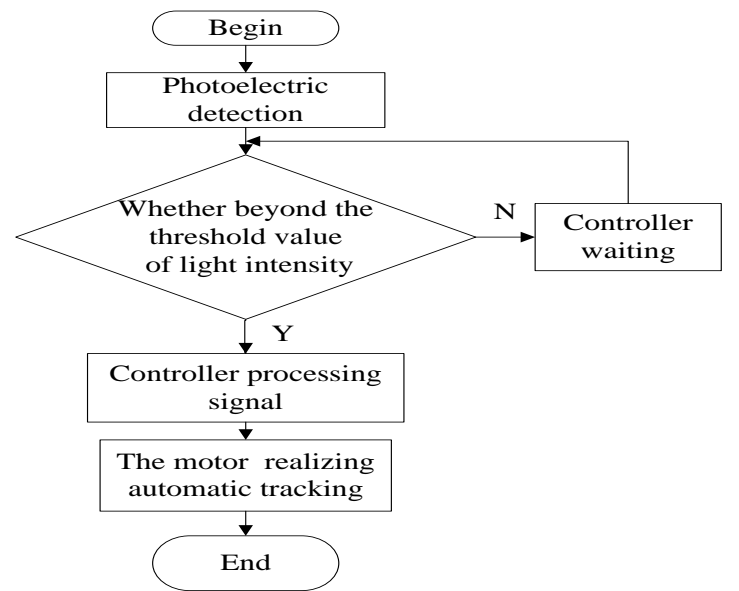

Fig. 7 process for the tracking of the source of the sun

\section{Comparison and analysis of the result of the experiment}

Nine-axis posture sensor is fixed on the solar panel and connected to the computer via the arduino core board. During the process of solar panel aiming at the light source, the values of Yaw, Pitch and Roll obtained by the MATLAB program are displayed in real time [8].Values of three variables for two schemes are shown in Figure 8.
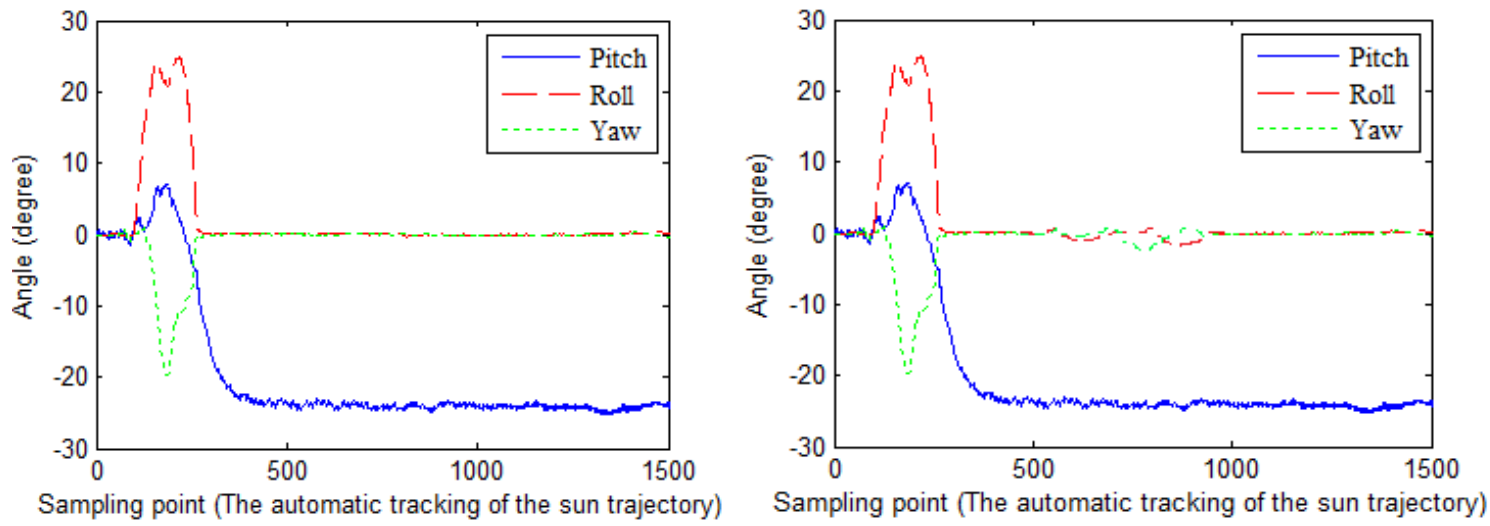

Fig. 8 values of three variables for two schemes

These conclusions are obtained from Figure 8:

a. Trajectory tracking scheme and photoelectric detection scheme can realize the basic function of the spotlights.

b. The automatic tracking scheme of the sun's trajectory can precisely track the sun's trajectory because it depends on precise calculation of the altitude angle and azimuth Angle of the sun, which can be used to send certain control signal to motor through the controller. The information of latitude, longitude and time is used as the control signal, so the light tracking system runs smoothly. But the model error exists.

c. In terms of automatic tracing scheme of sun light, when the light intensity is stronger, tracing scheme of the sun light runs more exactly than tracking scheme of the sun's trajectory .But in cloudy day or the day when light intensity is insufficient, the tracking system may be disordered.

Finally, the author combines two designs' merits together to overcome each other's drawbacks. Therefore, it turns to automatic tracing of solar light source when the light is strong, otherwise, it would tend to tracking scheme of the sun's trajectory. Such device, according to experiments, is afforded to tracking light precisely. 


\section{Acknowledgement}

This work was sponsored by Science and Technology Plan Project of Shenyang(No. F13-181-9-00), Science and Technology Plan Project of Shenyang(No.F13-316-1-74), the Chinese Defense Advance Research Program of Science and Technology, China under Grant (No. 62501040412),LiaoningBaiQianWan Talents Program(No.2013921069),Talent Resources Develop ment Special Funds of Shenyang(2010010403), The National High Technology Research and Development Program of China (No.2015AA042302).

\section{References}

[1] Chaofeng Xiao, Huilong Luo,Runsheng Tang. Solar thermal utilization in China[J].Renewable Energy,2004,29:1549-1556.

[2] Wencan Xu, Jun Yuan, Wei Yan. Research and experiment of automatic tracking solar energy system[J]. Physics Experimentation.2002, 23(9):45-48.

[3] Guoping Dai. Holl effect principle and application analysis[J].Science\&Technology Association Forum,2013(11):34-35.

[4] Tianwen Li. GPS Principle and Application[D].Beijing:Science Press,2003

[5] C.Thoms. Real-Time Restitution of GPS Time Through a Kalman Estimation[J].Metrologia, 2004(29):397-414.

[6] Hongmiao Lei,Yaoyu Cheng.Optimization design of DC motor drive circuit based on L298N[J]. Digital Technology\&Application.2012(2):118-120.

[7] Caihao Cui, Yuhua Zhang, Shucai Yang.Design of the light-guided car by using arduino control board[J]. Process Automation Instrumentation,2011,32(9):5-7.

[8] Dingyu Xun, Yangquan Chen.Advanced applied mathematical problem solutions with MATLAB (Third Edition)[D].Beijing:Tsinghua university press,2013. 